\title{
Optimization of Specimen Preparation Methods for Cryo Electron Microscopy of Oil- and-Water Emulsions
}

Dalaver H. Anjum ${ }^{*}$, Paolo Guida ${ }^{2}$, Ali R. Behzad ${ }^{1}$, Saumitra Saxena ${ }^{2}$ and William L. Roberts ${ }^{2}$

1. King Abdullah University of Science \& Technology (KAUST), Core Labs, Thuwal, Saudi Arabia.

2. Clean Combustion Research Center, King Abdullah University of Science \& Technology (KAUST), Thuwal, Saudi Arabia.

* Corresponding author: dalaver.anjum@kaust.edu.sa

Oil and water do not mix usually but can form emulsions i.e. they coexist as fluids containing separate phases of oil and water. Oil-and-water emulsions can be divided into two classes namely oil-in-water $(\mathrm{O} / \mathrm{W})$ and water-in-oil $(\mathrm{W} / \mathrm{O})$ emulsions. Both classes are important to several key industries including the extraction of crude-oil from wells and the combustion of crude-oil. Process of extracting crude-oil from wells involves water injection into reservoirs resulting in the formation of produced water (PW) which is basically $\mathrm{O} / \mathrm{W}$ emulsions [1]. Similarly, crude-oil prepared W/O emulsions are used to improve the combustion efficiency and reduce the environmental impact [2]. Three main phenomena are involved in the improvement of the combustion performance. The presence of water leads further oxidation of $\mathrm{CO}$ to $\mathrm{CO}_{2}$, reduces the average temperature in the combustion chamber reducing the $\mathrm{NO}_{\mathrm{x}}$ and generates the so called micro-explosions which basically consist in the eruption of supercritical water from the fuel which is further atomized in the spray enhancing the surface area and reducing the carbon waste [3].

A number of challenges exist for the effective uses of these emulsions. For instance, oil-droplets in PW may exist in as small sizes as few nanometers and therefore making the discharge or reuse of PW a challenge. Likewise, combustion efficiency of crude-oil not only depends on the fraction of water in W/O emulsion but also on the size of water-droplets that can exist in the range from micro to nanoscales as well. Therefore, in order to optimize this technology, a proper characterization of the emulsions is required [3]. Cryo electron microscopy (cryoEM) is an excellent choice to meet these challenges because it provides images containing spatial resolutions in the range needed during the size-characterization of these droplets. Therefore the objective of this study is to develop protocols for carrying out a successful cryoEM imaging-analysis of both $\mathrm{O} / \mathrm{W} \& \mathrm{~W} / \mathrm{O}$ emulsions.

A specific $\mathrm{O} / \mathrm{W}$ emulsion, having applications for $\mathrm{PW}$ reuse, was considered and was synthesized by mixing $\sim 1 \%$ crude oil in water and then stabilizing it with sodium dodecyl benzene sulfonate (SDBS) surfactant. Similarly, the heavy fuel oil (HFO 380) emulsified through sonication at a frequency of 20 $\mathrm{kHz}$. This was followed by $1 \mathrm{wt} \%$ addition of surfactant to the mixture to improve the stability of emulsions. Both cryoEM-modalities namely cryoSEM and cryoTEM were used to perform microscale and nanoscale imaging analysis of emulsions. A Nova NanoSEM equipped with cryo preparation chamber and cryo stage was used for cryoSEM work. Samples were prepared either by high-pressure freezing (HPF) or by plunge freezing of emulsions followed by fracture and subsequent sublimation in the cryopreparation chamber and cryoTEM-imaging was performed with Titan $\mathrm{G}^{2}$ Krios TEM. Cryo TEM samples were prepared using an automatic plunge-freezing system (Vitrobot Mark-IV).

Figure A shows a cryoSEM-image of $\mathrm{O} / \mathrm{W}$ emulsion after HPF, freeze fracture and subsequent sublimation. Only the oil droplets that were physically attached to the sample holder and remained attached after sublimation were imaged. Most of the oil droplets that were suspended in the water matrix were lost during the sublimation step and therefore the size measurements of the observed droplets do not 
provide actual statistics on the size of droplets. The loss of droplets during sublimation was prevented by adding $1 \%$ agarose to the emulsion mixture. A typical cryoSEM-image of agarose containing $\mathrm{O} / \mathrm{W}$ emulsion is shown in Figure B. Agarose helped in keeping the oil-droplets intact in the fractured plane after sublimation step and thus making the imaging and the size measurements possible. On the other hand, the plunge freezing, freeze fracture and sublimation of $\mathrm{W} / \mathrm{O}$ emulsion showed a number of empty holes in the fractured plane. The empty holes indicates presence of different size water droplets in the oil matrix (Figure C).

The O/W emulsion was found out to be quite a suitable sample for cryoTEM analysis and as shown in Figure D droplets of different size in nanometer range could easily be observed. However, cryoTEM on $\mathrm{W} / \mathrm{O}$ emulsion was not successful as indicated by its fuzzy image (Figure E). This fuzziness in the image implies that the prepared-specimen was too thick to image with cryoTEM. This was very likely due to high viscosity of $\mathrm{W} / \mathrm{O}$ emulsion. To reduce the emulsion viscosity a further $85 \%$ water was added to the stabilized W/O emulsion. Specimen from this less viscos $\mathrm{W} / \mathrm{O}$ emulsion showed higher quality cryoTEM images of the droplets (Figure F). Water-Oil droplets of W/O emulsion are marked with white-color circles (Figure F). Artifacts related to ice contamination (indicated by red circles) were also observed in both sample preparations (Figures $\mathrm{E}$ and $\mathrm{F}$ ) and happened during the transfer of specimens to microscope. In summary, optimization of specimen preparation method always holds the key to realizing a successful cryoEM imaging of both types of water-and-oil emulsions.

\section{References:}

[1] S Kumar et al., Journal of Petroleum Engineering \& Technology 5 (2015), p.1.

[2] Y Yan et al., Chemical Engineering and Processing 109 (2016), p. 90.

[3] L Feng et al., Energies 8 (2015), p. 13628.
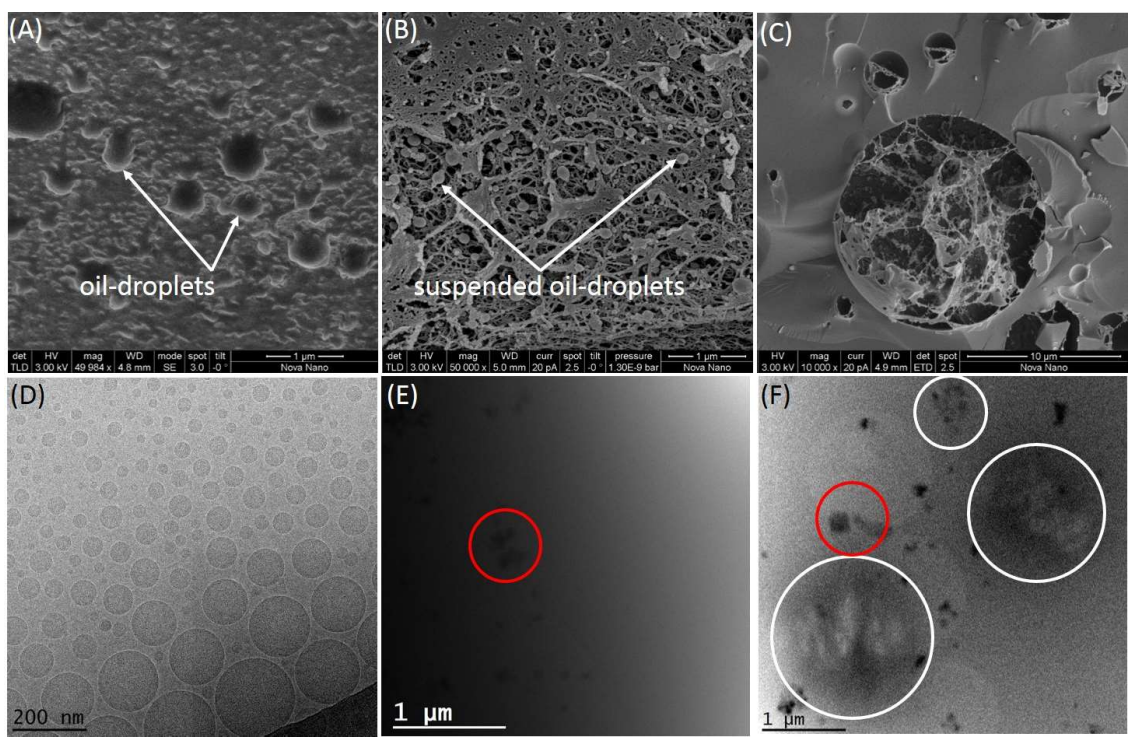

Figure 1. Imaging-analyses of $\mathrm{O} / \mathrm{W}$ and $\mathrm{W} / \mathrm{O}$ emulsions with cryoSEM and cryoTEM 